AGH DRILLING, OIL, GAS • Vol. 33 • No. $2 \cdot 2016$

http://dx.doi.org/10.7494/drill.2016.33.2.549

\author{
Monika Cepil*, Czesław Rybicki***
}

\title{
APPLICATION OF COMPACT SEPARATORS IN DEVELOPMENT OF NONCONVENTIONAL GAS RESERVOIRS****
}

\section{INTRODUCTION}

The separation relies on the separation in the effective way from each other two or three phases. The mechanism of the chapter of the gaseous phase from the oil phase is shown in Figure 1.

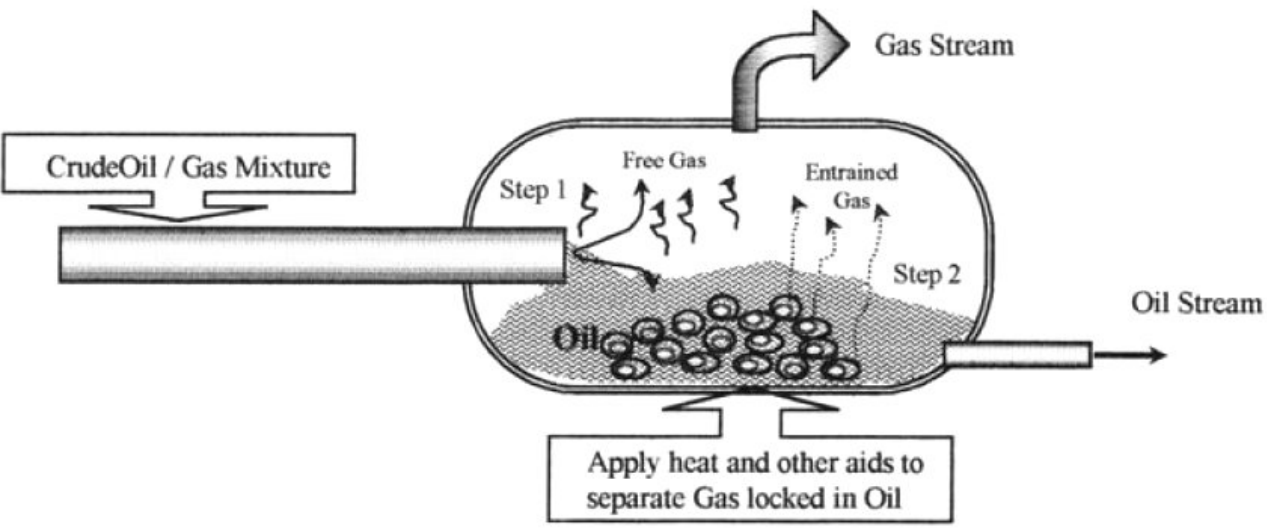

Fig. 1. The mechanism of phases divorce during the process of the separation [1]

* AGH University of Science and Technology, Faculty of Drilling, Oil and Gas, Krakow, Poland PhD Student

** AGH University of Science and Technology, Faculty of Drilling, Oil and Gas, Krakow, Poland

*** The article prepared within the framework of realized project "The elaboration of optimumconcepts of farm implements of unconventional deposits with the regard of aspects environmental and social" No. 3 Effective technologies of the preparation of gas to the transport from slate-formations of realized on WWNiG THE PROJECT REALIZED WITHIN THE FRAMEWORK OF THE PROGRAMME BLUE-GAS - POLANDS SLATE-GAS: BG1/ResDev/1 
As it gets out of the drawing (Fig. 1) in the process of the separation take part mainly two mechanisms. This are the gravitational separation appearing in the first instance and breaking free gas dissolved in oil in the second order. In the following part of the paper were discussed mechanisms of the separation

\section{ELEMENTS OF THE PHASIC EQUILIBRIUM OF THE SYSTEM LIQUID - IT ENGAGES}

The phase is defined, as the part of the system about equal physical and chemical properties, the homogeneous composition and separated from other phases with the terminal surface. Most important phases appearing in the production of hydrocarbons, which are oil and natural gas is the liquid phase and the gaseous (more seldom the solid phase-"hydrates") phase. Water is usually also the present, as additional liquid phase or in the form $\}$ of the gas. The term "of the pair" is used often interchangeably, as "gas", despite that proper his definition limits herself only to the area among the triple point and the critical point of the pure substance.

The diphase equilibrium liquid-gas (liquid-engages) in natural gas, and particularly dew-points of water and hydrocarbons belong to basic issues required in correct of his exploitation. That's why, conditions under which different phases remain in the equilibrium have the huge meaning in the obtainment of hydrocarbons, among other things at the designing of surface separators. These phases can remain in the equilibrium then, when variable describing the given equilibrium remain constant in time and space. Main variable defining the state of equilibrium are: the temperature of the system $T$, the pressure $P$ and the composition $z_{i}$. In connection with of above, very important is the settlement of temperatures and pressures for the given composition of the mixture for which phase equilibria they can appear. Equilibrium calculations are based on the concept of the constant equilibrium [1].

\section{MECHANISMS OF THE SEPARATION}

To the separate of multiphase systems of the type of gases-liquid or gas-liquid the solid one uses several types of mechanisms of the separation. This are among other things: the change of the sprout, the gravitational fall, coalescence and breaking free gas dissolved in phase liquid.

\subsection{The change of the sprout}

Moving components of the multiphase mixture have different magnitude of the sprout, what is tied with their different density. In case of the violent change of direction of the stream of the diphase medium, the sprout of particles of the lighter phase 
will permit the faster change of direction of the flow than particles of the heavier phase. This rule is used at the separation of mixtures trans fluent with the large expense.

\subsection{The gravitational fall}

Particles of liquid remain in gas, so long as is long the balance of power of the buoyancy, resisting forces and gravities. Gravities are directed down, forces of the buoyancy upward, however resisting forces toward opposite to the direction of the fall of the particle. All these forces have influence on the terminal rate of descent of the particle. On the equilibrium of these forces has influence the speed of transfluent gas. The essential meaning for the process of the fall of particles has the so called terminal speed of gas. This is such speed of below which begins the process of the fall of particles. The gravitational fall is described two established laws depending on the diameter of the drop and the Reynolds number. These laws are: Newton's law binding for drops about the greater diameter than $1000 \mu \mathrm{m}$ and Stokes' law applied for small drops about the diameter within the range from 3 to $100 \mu \mathrm{m}$. The range of particles among 100 and $1000 \mu \mathrm{m}$ is a transitory range where partly are in force both laws.

The terminal speed of the fall of particles of liquid $w_{k}$ one can enumerate from the following dependence:

$$
w_{k}=2 \sqrt{\frac{g\left(\rho_{l}-\rho_{g}\right) D_{k}}{3 \rho_{g} C}}
$$

where:

$w_{k}$ - the terminal rate of descent of particles,

$\rho_{l}-$ the density of liquid,

$\rho_{g}$ - the gas density,

$D_{k}$ - the diameter of the drop,

$C$ - the drag coefficient of the flow.

\subsection{Coalescence}

The coalescence phenomenon relies on the apartment of small drops into greater which can be separated thanks to the gravitation. So that this phenomenon be effective one ought to aim to this so that tracks of molecules so run it would come to collisions between them.

\subsection{Breaking free gas dissolved in phase liquid}

As is known the quantity of dissolved gas in phase liquid depends on the pressure and the temperature. If the pressure drops, the quantity of dissolved gas falls. Therefore essential it is to release maximally gas dissolved earlier in oil one ought to lower the pressure and to maximize the surface of the separation of phases. 


\subsection{The operation of the centrifugal force}

With the additional mechanism which maybe to influence the efficiency of the separation of particles is the operation of the centrifugal force. This mechanism relies on disordering the rotary motion of the multiphase mixture. In this result arises the centrifugal force causing that particles of the heavier phase are thrown out toward the side of the separator, where reaches the coalescence phenomenon and the gravitational fall after the side of the separator. From research and analyses led in different centers it results that separators using the centrifugal force gather the more and more wider use. Is developed in this range the construction of compact separators whose operation chiefly is based on the use of the centrifugal force.

\section{THE SUPERFICIAL SEPARATION}

The deposit fluid after the extraction from the deposit and to the possible auxiliary processing (e.g. the drainage) is subjected to the process of the separation. This process targets the separation from each other light and heavy hydrocarbon fractions, in as maxi-mum quantity. Heavy oils are subjected most often to the process of the two-grade separation, however light oil and condensate gas to the process of the three-stage separation [4].

It is known, that to the emission himself gas from liquid reaches in the moment, when the pressure will drop to the pressure of the saturation. Further his fall causes the growth of the extraction of gas. Higher described effect can reach in the deposit during the inflow of oil to the bore-hole or during the exploitation in mining pipes. Usually to the separator swims the diphase system liquid-gas whose then the mutual volumetric relation it depends on the composition of chemical gas and oil, and also on the pressure and the temperature.

In connection with of above in the separator in already one mentioned earlier will reach two main processes:

- separately free gas from liquid,

- the gas evolution of dissolved in liquid consequently the further pressure drop [7].

In practice industrial complies the preliminary separation "of the open water" (the water fraction obtained as the result of the gravitational settlement) before the accession to the proper cleaning of remaining oil and the layer of the emulsion. For that purpose it complies separators three-phase, called sometimes with separators "of the open water" which separates this and remove the entire water phase [7].

\subsection{Gravitational separators}

Gravitational separators in respect of the shape and the location it divides into separators cylindrical (vertical or horizontal) and spherical. 


\section{The diphase vertical separator}

In the vertical separator (Fig. 2) the fluid inflowing to its through the inlet meets the element breaking the stream, what gives rise to the chapter of liquid and gas. If at the given temperature and pressures the fluid achieves the state of equilibrium, then being emitted bubbles of gas rise to the head of the separator toward opposite to the stream of liquid and achieve the space of the gaseous phase.

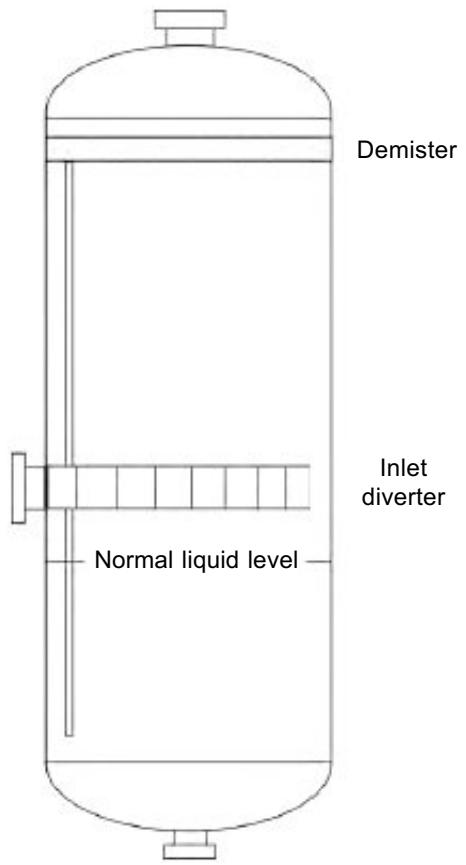

Fig. 2. The diagram of the diphase vertical separator [3]

\section{The diphase horizontal separator}

In Figure 3 is presented the typical example of the diphase horizontal separator. The inflowing fluid to the separator meets on its way the element causing the sea change of his speed and the direction of the flow, consequently one initiates the separation of liquid from gas. Heavier droplets of liquid, under gravitate pulls, fallout from the gas current and assemble on the bottom of the separator. The section of the accumulation of liquid stops the liquid phase during the reasonable time of the retainer of permitting dispersed, with the particle of gas to separate from oil and to form the area par. After the separation liquid sails away from the separator with the automatic valve which this is corrected a regulator of the level of liquid [7]. Horizontal separators I can have they the any length, however the relation of their length (counted from the seam to the seam to the diameter is usually in the compartment $2: 1$ to $6: 1$. Separators working at greater 
pressures from economic favor are designed as more slender [1]. The lissomness of these separators gets out of the fact that to them the higher gas pressure this less this gas is in respect of volume.

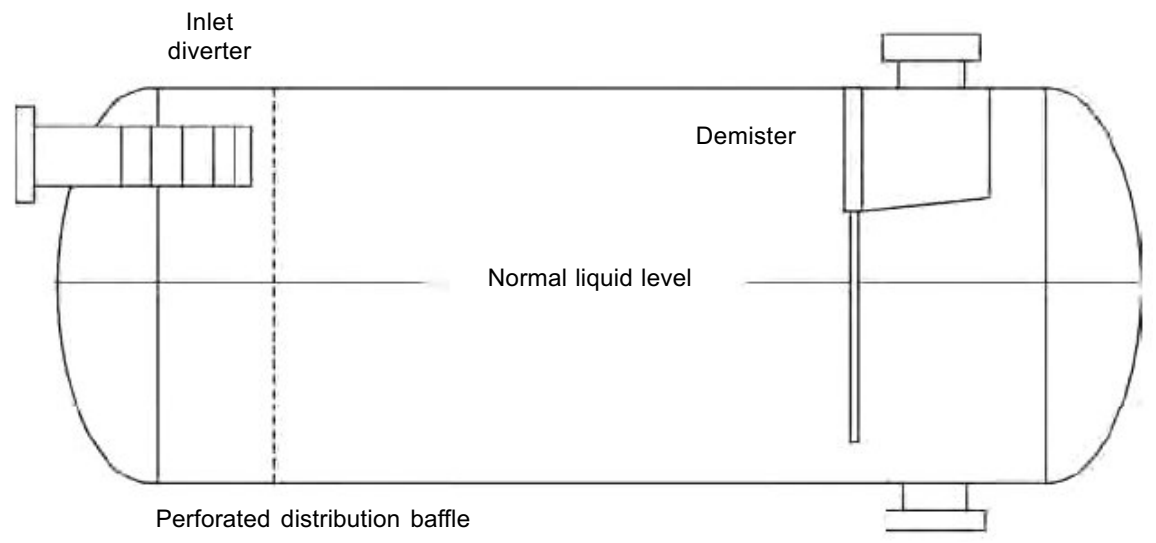

Fig. 3. The construction of the horizontal separator [3]

Horizontal separators characterize themselves [3]:

- with the large interphase surface,

- long sometimes the retainer of liquid,

- with the dependence of the speed from the height of the level of liquid,

- with low costs,

- with the simple service.

\section{The spherical separator}

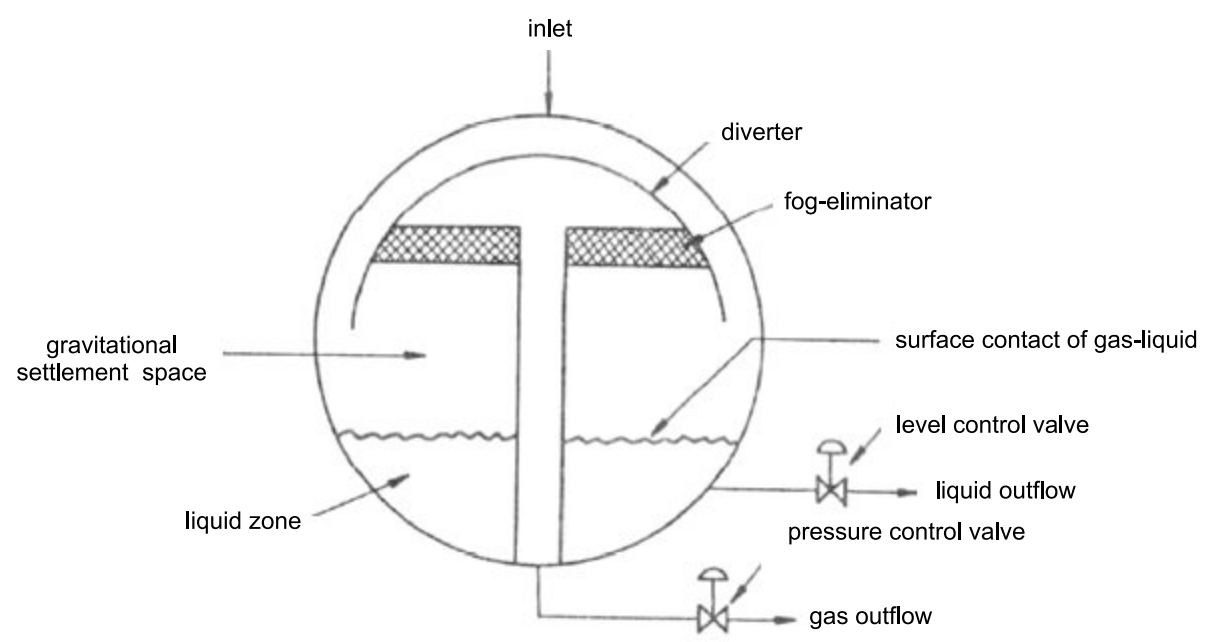

Fig. 4. The diagram of the spherical separator [2] 
In spherical separators (Fig. 4) like in vertical and horizontal, one can separate four sections:

- The section breaking the stream on the inlet.

- The section of the gravitational settlement.

- The section of the accumulation of liquid.

- The section of the eliminator of the fog of liquid [7].

\section{COMPACT SYSTEMS OF THE SEPARATION}

This part of the article is sacrificed to the discussion of most important aspects related to the compact system of the separation of hydrocarbons. Were described elements of the construction of one of compact separators, namely so called GLCC (Gas/Liquid Cylindrical Cyclone), and also the idea of his work and the use in the oil industry.

\subsection{The genesis of the development of compact systems of the separation}

In the past the process of the separation, the multiphase flow from the reservoir, in the oil industry was based mainly on conventional, large-size separators. However aspects economic and operational obliged the industry to the search of cheaper and more effective methods of the separation. From the early 90 s of the $20^{\text {th }}$ century, the oil industry sacrificed many attention to compact separators, too such as Gas/Liquid Cylindrical Cyclone (GLCC).

\subsection{The construction and geometric aspects of the separator GLCC}

Laboratory tests and computer simulations showed with as essential aspect inflowing on the efficiency GLCC is the configuration, the size and the shape of his individual elements. Despite the relatively simple construction of the separator GLCC, geometric aspects have a very essential influence on the process of the separation. Measurements of this separator influence also costs of the process [8].

The separator GLCC presented in Figure 5 has the form of the vertical cylindrical pipe. Considerations over the system of walls of the cyclone GLCC showed, that cylindrical their position is somewhat better for the process of the separation, than their convergence or the divergence.

As it is visible from the Figures 5 and 6 the separator GLCC has the tangent, sloping inlet and the outlet in the head for gas and the foot for liquid. The diameter, the depression angle and the length are key-parameters of the sloping admission pipe. The outlet of gas and liquid should have the suitable inside diameter providing required for the separation the efficiency, and also guaranteeing the elimination of undesirable phenomena, such as the choke in the gaseous outlet [10]. 


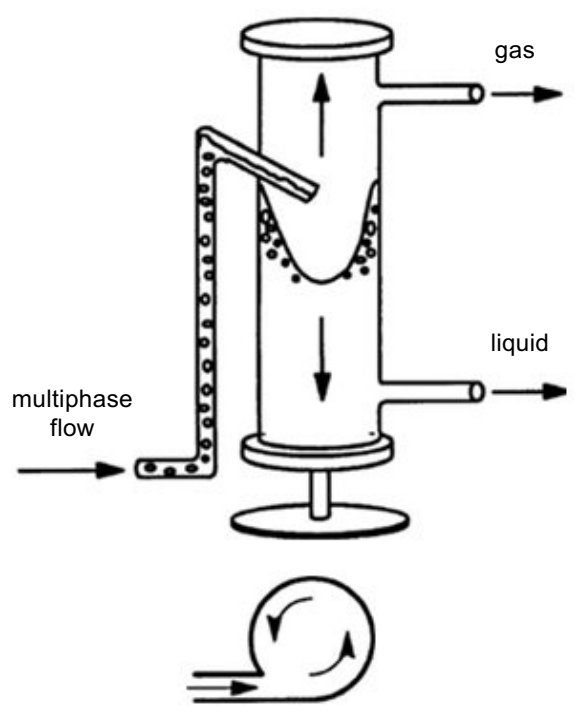

Fig. 5. Visual diagram of the separator GLCC [1]

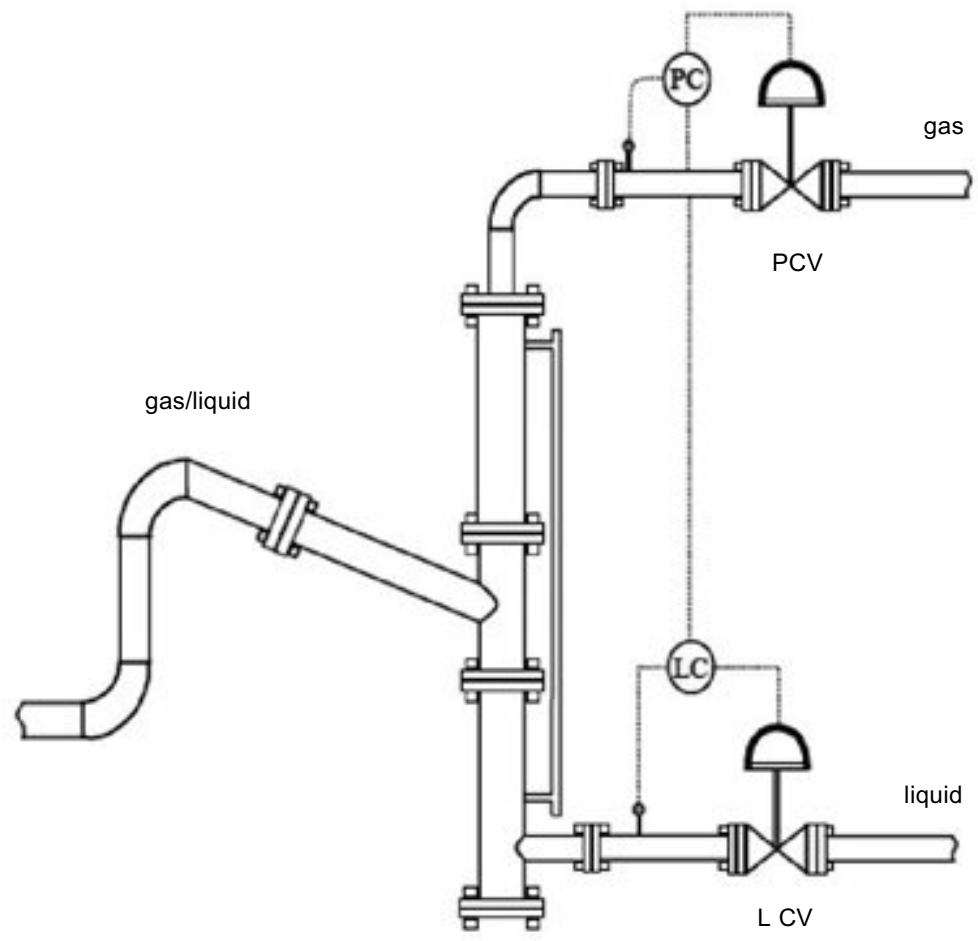

Fig. 6. The construction of the separator GLCC [6] 
In conventional separators the inlet of the fluid to the separator is usually perpendicular what also confirms the review of the literature. However investigations over GLCC showed that the inclination of the inlet improved the efficiency of the process of the separation across reductions of the undesirable phenomenon so called liquid carryover. Compact GLCC is deprived mobile parts and internal elements.

The inlet nozzle, that is the last element of the sloping admission pipe, influences the schedule of the flow and the intake-speed, because reduced area of the nozzle causes the acceleration of the diphase stream.

The tangent nozzle is dearest to producing a part GLCC. For the purpose of the optimization of parameters of the flow were tested numerous configurations of nozzles which additionally were supposed to fulfil a condition of their effective economically the production. An optimum-, hydro dynamically efficient configuration turned out the thin nozzle with the rectangular inlet to the corps GLCC. It is however difficult to the production. One fixed that the concentric circular inlet was easier in the production, but shows the lower efficiency. The preliminary, experimental comparison of three shapes of the intake-slit: rectangular, concentric of round and crescent-shaped, about the same sectional area, it found that the concentric round nozzle had had worst results, while the moon-shaped nozzle showed nearest results to the rectangular inlet [10].

Laboratory tests and computer simulations showed with as essential aspect influencing on the efficiency GLCC is its construction, and what this is going the configuration, the size and the shape of his individual elements. Despite the relatively simple construction of the separator GLCC, geometric aspects have a very essential influence on the process of the separation. Measurements of the separator influence also costs of the process [11]. In Figure 7 one showed the comparison of measurements of the separator horizontal, vertical and the low sized separator GLCC.

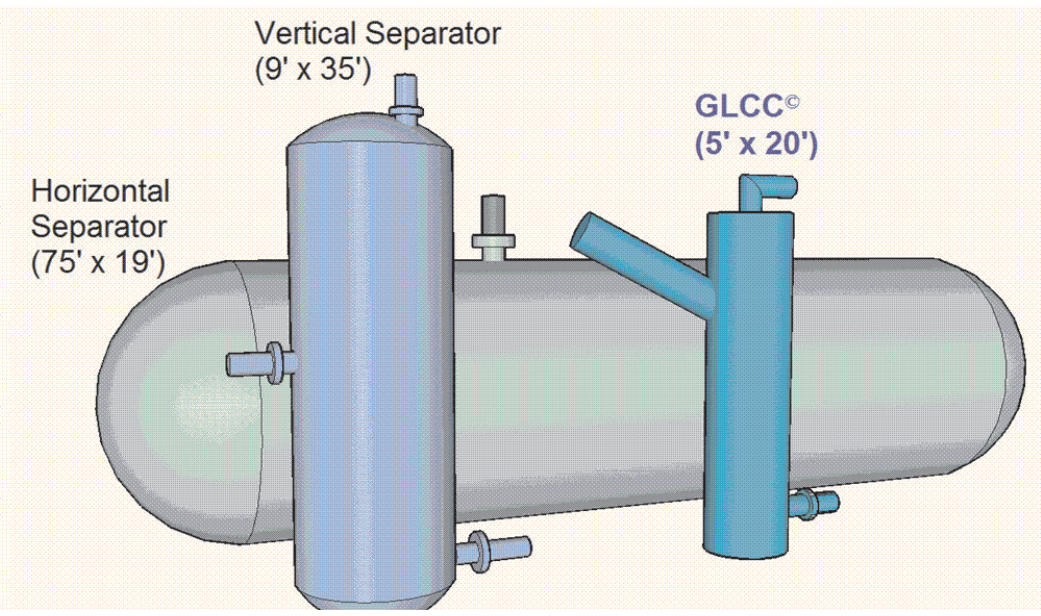

Fig. 7. The comparison of the size of the separator GLCC, vertical and horizontal $[7,5]$ 
GLCC has the tangent, sloping inlet and the outlet in the head for gas and the foot for liquid. The diameter, the depression angle and the length are most important parameters of the sloping admission pipe. Compact GLCC does not have mobile parts and internal elements.

\section{PHENOMENA OF "LIQUID CARRY OVER" AND “GAS CARRY UNDER"}

As it gets out of led research sizes of the separator GLCC determine additionally two limiting phenomena. One of them is the phenomenon of the carriage of liquid in the gas current so called liquid carry-over and the phenomenon of the transfer of gas in the stream of liquid, so called gas carry-under.

Liquid carry-over is considerably dependent from the diagram of the flow in the head of the corps GLCC. To the mentioned effect it can come at high levels of liquid and low gas flows, what causes the coming into being of the dispersed blisters of gas in the continuous liquid phase flow. At the growth of the expense bubbles of gas join in greater blisters so called Taylor blisters. Among large blisters of gas appear corks of liquid with smaller blisters of gas. At the further growth of the expense of gas large blisters of gas the furlong themselves perishable and both phases are dispersed. Unstable liquid oscillations can inject the fluid to the inlet of gas. At still higher expenses of gases it becomes a continuous phase with dispersed drops of liquid. The centrifugal force of gas pushes liquid to sides of the corps GLCC, creating continuous the band of liquid [10, 8]. With the sentence of authors presented separator GLCC is only one of possible solutions of new technologies to the separation of gas. The use of new parts of modular installations would be able to permit on the restriction of the place, costs and first of all would improve the gas transportation system.

\section{CONCLUSIONS}

1. On deposits about a very small permeability the efficiency of wells quickly decreases, therefore purposeful would be the compliance of separators about small measurements and the high performance.

2. Such conditions fulfil centrifugal separators of the type GLCC.

3. A sentence of authors purposeful \{intentional $\}$ is designing and the construction of modular separator which will constitute the element of the set to the separation.

4. In the initial operating period into the composition of the separation system would enter for example several in parallel connected separators which the measure of the period of time would be excluded from the exploitation. Such solution will ensure high efficiency of the process at the cost minimization. 


\section{REFERENCES}

[1] Gomez L.E. et al.: Enhanced Mechanistic the Model and Field-Application Design of Gas-Liquid Cylindrical Cyclone Separators. SPE 49 174, 1998.

[2] Jewulski J.: Napowierzchniowe zagospodarowanie złóż surowców płynnych. Minerals, Cracow, AGH 2005.

[3] Arnold K.E. (Ed.): Facilities and Construction Engineering. Vol. 3. In: Larry W.L. (Ed.), Petroleum Engineering Handbook. SPE Richardson 2007.

[4] Molenda J.: Gaz ziemny. WNT, Warszawa 1974.

[5] Molina R.C.: Wet Gas Separation in Gas Liquid Cylindrical Cyclone (GLCC@) Separator. University of Tulsa, 2003.

[6] http: //www.t ustp.org/ (access 10.02.2016).

[7] http://tustp.org/publications/integ_level_pressure_control_00.pdf (access 17.08.2016).

[8] http://tustp.org/publications/state of art tech 98.pdf (access 05.01.2016).

[9] http://msieng.com/pages/sub/other/glcc.htm\#adv (access 10.02.2016).

[10] http://tustp.org/publications/enhanced mechanistic the model 98.pdf (access 02.01.2016).

[11] http://www.accuflow.com/ (access 03.01.2016).

[12] http://tustp.org/publications/review glcc tech 96.pdf (access 03.01.2016). 